\title{
Event-Based Integral Control for Output Disturbance Rejection
}

\author{
Fairouz Zobiri*†, Nacim Meslem*, and Brigitte Bidegaray-Fesquet ${ }^{\dagger}$ \\ *Univ. Grenoble Alpes, CNRS, Grenoble INP $\ddagger$, GIPSA-lab, 38000 Grenoble, France \\ ${ }^{\dagger}$ Univ. Grenoble Alpes, CNRS, Grenoble INP ${ }^{\ddagger}$, LJK, 38000 Grenoble, France \\ $\ddagger$ Institute of Engineering Univ. Grenoble Alpes \\ Emails: Fairouz.Zobiricuniv-grenoble-alpes.fr, \\ Nacim.Meslem@gipsa-lab.grenoble-inp.fr, \\ Brigitte.Bidegaray@univ-grenoble-alpes.fr
}

\begin{abstract}
In this article, we treat the problem of disturbance rejection in event-based control. We consider the case of an LTI system under the influence of an output disturbance. We base our event-triggering conditions on the comparison between the actual system and a fictitious reference system with an ideal behavior. However, since we do not know the value of the disturbance, this comparison is made difficult. So, we propose a solution inspired from fault-diagnosis methods where we build an online estimator to estimate the value of the disturbance. We provide, then, this information to the reference system and proceed with comparing the states of the two systems.
\end{abstract}

Keywords-Event-based control; output disturbance; state observer; fault-diagnosis; integral action.

\section{INTRODUCTION}

In classical control theory, sensors measure system parameters continuously and a continuous control law is calculated and applied. On digital platforms, the control law is translated into a periodic, oversampled signal that is applied even when no action is needed. A more flexible alternative is clearly necessary.

Event-based control has come to light as such an alternative. This approach consists in computing and updating the control law only when a set of predefined conditions on the behavior of the system is violated. These conditions are generally related to the state of the system and the quality of its response. Among the first works on event-based control, we can cite [1] in which an event-based counterpart for the PID controller is designed. Many others have followed afterwards, such as [2], [3], [4], [5], and [6]. This method has the advantage of decreasing the communications between the CPU and the actuators, therefore avoiding the congestion of the communication channels. However, such benefits do not come easily and many challenges arise. There is primarily the problem of the choice of the adequate event-triggering conditions that ensure stability and performance of the system despite the fact that it is controlled only intermittently. By using this approach, we also need to find a trade-off between the frequency of communication and the performance of the system.

In reality systems are subjected to the influence of load disturbances. Thus, we clearly want our event-based approach to be robust enough to the impact of the surroundings on the system. Disturbance rejection has been explored in a few previous works. In [7], an upper bound on the disturbance is used as the event-triggering condition. In [8], an augmented observer is built in order to estimate the state and the disturbance simultaneously. A composite controller is then designed to compensate the disturbance actively. In [9], it is the issue of model uncertainties that is addressed. Recently in [10], an observer-based approach has been proposed to deal with the state disturbance rejection problem with an event-triggered mechanism. To do that, the proposed approach assumes that the considered disturbance and its derivative are bounded.

In this work we explore a procedure for the event-based control of a linear time invariant (LTI) system. Unlike to [10], the objective is to drive the system to follow a predefined trajectory in the presence of an unknown low-frequency output disturbance. As in [11], the state of the system is compared to the state of a continuously-controlled reference system. But here, the disturbance renders this comparison difficult since it is a priori unknown to the reference system.

For this, we build an estimator made of two series blocks (see [12]). The first block is an observer that provides the residual between the observed and the measured output of the event-based system. The second block consists in a filter that estimates the value of the disturbance. This information on the disturbance is, then, given to the reference system. Finally, an integral action is added to ensure the rejection of the disturbance.

This work is divided as follows. The next section is dedicated to the description of the system to be controlled, the reference system and the disturbance estimator. It also introduces the event-based conditions and how they are built. In Section III a discussion on the performance of the system is presented. Finally, a numerical example is treated in Section IV.

\section{DESCRIPTION OF THE SYSTEM}

\section{A. Overall principle}

We consider a Linear Time-Invariant system controlled to follow a predefined reference signal $r(t) \in \mathbb{R}^{n_{y}}$ and to reject the effect of an additive disturbance $d(t) \in \mathbb{R}^{n_{y}}$. This system is driven with an event-based state-feedback control $\bar{u}(t)$ which is piecewise constant. This control law is augmented by an integral action to cope with the output disturbance.

The times $t_{k}$ when the control is updated is event-based and corresponds to the detection of the fact that the system drifts away from a reference system. Contrarily to the real system, the reference one is controlled continuously and does not see the disturbance. However, we need to estimate this disturbance. This is performed by introducing a third system, an online disturbance estimator, which has only a knowledge of the event-based control and the output of the real system, and that feeds the reference system with the estimated disturbance 


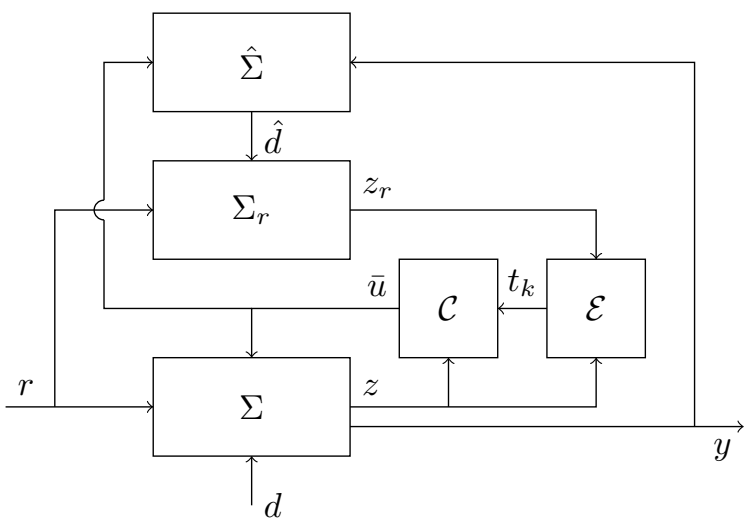

Figure 1. The event-based controlled system $\Sigma$, the reference system $\Sigma_{r}$, the estimator $\hat{\Sigma}$ and the event generator $\mathcal{E}$

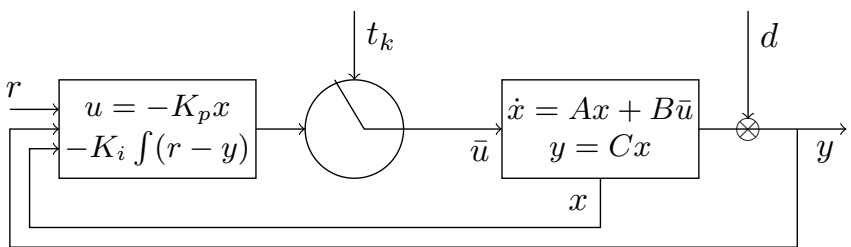

Figure 2. A detailed layout of the signals involved in the event-based controlled system

$\hat{d}(t)$.

Fig. 1 and 2 are schematic diagrams of the approach detailed above. More precisely, Fig. 1 displays the event-based controlled system $\Sigma$, the reference system $\Sigma_{r}$, and the disturbance estimator $\hat{\Sigma}$. Comparing the augmented states $z$ and $z_{r}$ of systems $\Sigma$ and $\Sigma_{r}$ respectively, the event generator $\mathcal{E}$ evaluates the event times $t_{k}$ at which the controller $\mathcal{C}$ should compute a new control value $\bar{u}$.

\section{B. An event-based augmented system}

1) The LTI system and exogenous signals: The LTI system is given in the state-space representation by

$$
\begin{aligned}
& \dot{x}(t)=A x(t)+B \bar{u}(t), \\
& y(t)=C x(t)+d(t) .
\end{aligned}
$$

In this system $x(t) \in \mathbb{R}^{n_{x}}$ is the state vector, $y(t) \in \mathbb{R}^{n_{y}}$ is the system output and $\bar{u}(t) \in \mathbb{R}^{n_{u}}$ is the system input. The system is assumed to be controllable. Note that, in this context, a part of the output vector is assumed to belong in the state vector.

We suppose that the disturbance, as well as the predefined reference signal $r(t)$ are piecewise Lipschitz and uniformly bounded, and we authorize them to contain jumps, provided that there exists a minimum interval of time between two successive jumps.

2) Augmenting the system for tracking: The state vector $x(t)$ is augmeAnted to take into account an integral action on the tracking error

$$
\dot{\omega}(t)=r(t)-y(t) .
$$

This yields a system on the augmented state variable $z(t)=$ $(x(t), \omega(t))^{T}$

$$
\begin{aligned}
& \dot{z}(t)=\left(\begin{array}{cc}
A & 0 \\
-C & 0
\end{array}\right) z(t)+\left(\begin{array}{c}
B \\
0
\end{array}\right) \bar{u}(t)+\left(\begin{array}{c}
0 \\
r(t)-d(t)
\end{array}\right) \\
& y(t)=\left(\begin{array}{lll}
C & 0
\end{array}\right) z(t)+d(t) .
\end{aligned}
$$

3) Event-based triggering: A classical feedback control for this augmented system consists in defining a continuous control

$$
u(t)=-K_{p} x(t)-K_{i} \omega(t)=-K z(t) .
$$

Combing (4) and (6) would lead to the equation

$$
\dot{z}(t)=\left(\begin{array}{cc}
A-B K_{p} & -B K_{i} \\
-C & 0
\end{array}\right) z(t)+\left(\begin{array}{c}
0 \\
r(t)-d(t)
\end{array}\right) .
$$

The feedback control is then constructed such that the augmented matrix

$$
A_{a}=\left(\begin{array}{cc}
A-B K_{p} & -B K_{i} \\
-C & 0
\end{array}\right)
$$

is Hurwitz with desired eigenvalues. This matrix will be used to construct the reference system.

But in the event-based system $\bar{u}$ is the event-based counterpart of $u$. Its value is only changed at times $t_{k}$ with the following formula

$$
\bar{u}(t)=u\left(t_{k}\right) \text { for } t \in\left[t_{k}, t_{k+1}\right),
$$

and equation (4) is only valid at times $t_{k}$ after the control has been updated.

\section{The reference system}

The reference system is also an augmented system with an integral action to be able to compare the augmented state variable $z(t)$ to the reference state $z_{r}(t)$. The control (6) is computed continuously with the same approach as for (8) and we have $u_{r}(t)=-\left(K_{p} K_{i}\right) z_{r}(t)$ for all time $t$. The equation dynamical for $z_{r}$ is therefore

$$
\dot{z}_{r}(t)=\left(\begin{array}{cc}
A-B K_{p} & -B K_{i} \\
-C & 0
\end{array}\right) z_{r}(t)+\left(\begin{array}{c}
0 \\
r(t)
\end{array}\right) .
$$

If we only do this, thanks to the Hurwitz nature of $A_{a}$, the reference system follows the reference signal $r(t)$ but it is unable to send correct event-based controls (i.e. control times) to the real system in order to reject the disturbance. In practice, the distance between $z(t)$ and $z_{r}(t)$ which are respectively solution to (4) and to (9) has no reason to vanish, due to the presence of $d(t)$. From a certain time on, this difference would always be large, leading the event-generator to produce events each time the difference is evaluated, and the real system would then be a classical discrete-time controlled system and not an event-based one.

To solve this problem, we introduce an estimator that evaluates the disturbance acting on the output of the system. The estimated disturbance is then included in the dynamics of the reference system as follows

$$
\dot{z}_{r}(t)=\left(\begin{array}{cc}
A-B K_{p} & -B K_{i} \\
-C & 0
\end{array}\right) z_{r}(t)+\left(\begin{array}{c}
0 \\
r(t)-\hat{d}(t)
\end{array}\right) .
$$




\section{The disturbance estimator}

For sake of simplicity, and since the point here is to reject the disturbance and not show how to follow the reference signal, we suppose here that $r(t)=0$, but experiments in the numerical illustration section are performed with a nonvanishing reference signal.

To estimate the disturbance, we introduce a Luenberger observer assuming that $(A, C)$ is an observable pair. The observed state $\hat{x}$ is solution to the system

$$
\begin{aligned}
& \dot{\hat{x}}(t)=A \hat{x}(t)+B \bar{u}(t)+L(y(t)-\hat{y}(t)), \\
& \hat{y}(t)=C \hat{x}(t) .
\end{aligned}
$$

We notice that to write this observer we only need to know the basic elements of the LTI system (namely matrices $A, B$ and $C$ ), the output of the real system $y(t)$ and the event-based control $\bar{u}(t)$ (but not the way to compute it, which is only known by the controller). Once more the gain matrix $L$ is chosen such that $A-L C$ is Hurwitz, which ensures the stability of the observation error. From the knowledge of the difference $\tilde{y}(t)=y(t)-\hat{y}(t)$, using a Laplace transform analysis and Woodbury matrix identity, we classically obtain an estimated $\hat{d}$ which is the output of the linear filter

$$
\begin{aligned}
\dot{m}(t) & =A m(t)+L \tilde{y}(t), \\
\hat{d}(t) & =C m(t)+\tilde{y}(t)
\end{aligned}
$$

(see Appendix for details). The system composed of equations (13) and (14) is initialized at $m\left(t_{0}=0\right)=0$.

\section{E. Events}

The events are generated when the real state and the reference state are too far apart. Let $e(t)$ be the difference

$$
e(t)=z(t)-z_{r}(t)
$$

The sequel is very similar to the arguments used in [11]. Two symmetric positive definite matrices $P$ and $Q$ are defined via the Lyapunov equation $A_{a}^{T} P+P A_{a}=-Q$. The specification on the tracking error is then monitored via the Lyapunov-like function

$$
V(e(t))=e(t)^{T} P e(t) .
$$

A small constant $\delta$ is defined and the event times when the control is updated are given by the following definition.

Definition 1: The time $t_{k}$ is defined as the minimum time $t>t_{k-1}$ for which $V(e(t))=\delta$ :

$$
t_{k}=\inf \left\{t>t_{k-1}, V(e(t))=\delta\right\} .
$$

\section{Discussion}

From (16) we immediately infer that estimating $V(e(t))$ is also estimating $e(t)$ in the $L^{2}$-norm, indeed we have

$$
\sqrt{\frac{V(e(t))}{\lambda_{\max }}} \leq\|e(t)\| \leq \sqrt{\frac{V(e(t))}{\lambda_{\min }}},
$$

where $\lambda_{\min }$ and $\lambda_{\max }$ are the (real and positive) minimum and maximum eigenvalue of $P$. Hence to ensure that $\|e(t)\|$ is below some given $\varepsilon$, we simply set $\delta=\varepsilon^{2} \lambda_{\min }$ in Definition 1 .
We can rewrite (4) in an equation involving the augmented matrix $A_{a}$ and, combining with (10), we have for all time $t \in\left[t_{k}, t_{k+1}[\right.$

$$
\dot{e}(t)=A_{a} e(t)+\left(\begin{array}{c}
B K \\
0
\end{array}\right) \Delta_{k} z(t)-\left(\begin{array}{c}
0 \\
\tilde{d}(t)
\end{array}\right),
$$

where $\Delta_{k} z(t)=z(t)-z\left(t_{k}\right)$ and $\tilde{d}(t)=d(t)-\hat{d}(t)$.

We have already seen in [11] how to handle the $\Delta_{k} z$ term: despite the possible jumps in the reference signal $r(t)$ and in the disturbance $d(t)$, the states $z(t)$ and $z_{r}(t)$ are Lipschitz. In particular, there exists a constant $L_{z}$ such that

$$
\left\|\Delta_{k} z(t)\right\| \leq L_{x}\left(t-t_{k}\right) \text { for all } t \in\left[t_{k}, t_{k+1}[.\right.
$$

Regarding $d$ and its estimate, the situation is twofold. Either the initial data are well chosen (i.e. $m(0)=0$ and $\hat{x}(0)=x(0))$ and we have exactly $\hat{d}(t) \equiv 0$ for all time. In our numerical simulations below, for which a very unsophisticated, namely forward Euler, scheme has been used, if these initial conditions are fulfilled $\tilde{d}$ is zero up to the machine epsilon. If the right initial conditions are not taken, the observer and estimator yields a different value for $\hat{d}$ (for example in the permanent regime in the step disturbance test case) follows a bad track, $r+\tilde{d}$ instead of $r$. With convenient initial values we simply have

$$
\dot{e}(t)=A_{a} e(t)+\left(\begin{array}{c}
B K \\
0
\end{array}\right) \Delta_{k} z(t),
$$

and the estimates on $\left\|\Delta_{k} z(t)\right\|$ are sufficient to state the following results.

\section{A. Error estimate}

Theorem 1: If the triggering times are defined by Definition 1, the Lyapunov-like function $V(e(t))$ remains bounded by $\delta$.

At time $t_{k}$, after the update, we simply have $\dot{e}\left(t_{k}\right)=$ $A_{a} e\left(t_{k}\right)$ and

$$
\dot{V}\left(e\left(t_{k}\right)\right)=-e\left(t_{k}\right)^{T} Q e\left(t_{k}\right) .
$$

Since $V\left(e\left(t_{k}\right)\right)=\delta$, (17) yields a lower bound for $e\left(t_{k}\right)$ and a negative upper bound for $\dot{V}\left(e\left(t_{k}\right)\right)$ which ensures that $V(e(t))$ is pushed below $\delta$ for a certain amount of time. This proves theorem 1. As a corollary, $\|e(t)\|$ remains below $\varepsilon$.

\section{B. Minimum inter-sample delay}

The system is event based, because it is possible to derive from the parameters of the equation a minimum time delay between two updates of the control.

Theorem 2: The event times given by Definition 1 are at least spaced $\tau_{\min }$ apart from each other, i.e.

$$
t_{k+1}-t_{k} \geq \tau_{\min } \text { for all } k \in \mathbb{N} .
$$

This result can be proved exactly as in [11], using Equation (20) and the estimate (19).

The boundedness and inter-event time features are displayed in the numerical test cases of the next section. 


\section{Observing the states to define events}

Despite the availability of the estimated state $\hat{x}(t)$, we have been using the measured state $x(t)$ to monitor the performance of the system, to generate the events and to compute the control law. This is due to the presence of the disturbance, which makes it impossible for $\hat{x}(t)$ to converge to $x(t)$ as shown below. Let $\tilde{x}(t)$ be the observation error with dynamics

$$
\dot{\tilde{x}}(t)=\dot{x}(t)-\dot{\hat{x}}(t)=(A-L C) \tilde{x}(t)-L d(t) .
$$

Even if the observation error is stable, as $A-L C$ is Hurwitz, it never tends to zero, as long as the disturbance is present. This in fact is what allows us to estimate the disturbance. The only way to estimate the state is to have some prior knowledge of the disturbance. For instance some of the methods given in [13] assume that the disturbance is described by some known mathematical model.

In our case, when the disturbance does not obey any mathematical model, the only way to recover the state vector from the output of the system is to be able to measure the disturbance.

\section{Simulation Results}

\section{A. Step disturbance}

To validate the results detailed above, we test our approach on a study case.

Let us consider the linearized and simplified model for controlling the pitch angle of an aircraft given in [14] . The state-space matrices governing the motion are

$$
\begin{gathered}
A=\left(\begin{array}{ccc}
-0.313 & 56.7 & 0 \\
-0.0139 & -0.426 & 0 \\
0 & 56.7 & 0
\end{array}\right), B=\left(\begin{array}{c}
0.232 \\
0.0203 \\
0
\end{array}\right), \\
C=\left(\begin{array}{lll}
0 & 0 & 1
\end{array}\right) .
\end{gathered}
$$

In the simulation, we choose the entire simulation time $T_{\text {sim }}=20 \mathrm{~s}$ and the sampling period $\Delta t=10^{-4} \mathrm{~s}$ for a total number of $N=200,000$ sampling instants.

We want to control the pitch to hold a specific angle while being subjected to an external output disturbance. We set the reference signal and disturbance at

$$
\begin{aligned}
& r(t)=10 \Pi_{0,10}(t)+2 \Pi_{10,20}(t), \\
& d(t)=2 \Pi_{5,20}(t),
\end{aligned}
$$

where $\Pi_{a, b}$ is the boxcar function, which is equal to 1 on interval $[a, b]$ and zero otherwise.

In Fig. 3, the output of the system and the reference signal are depicted along with the estimated value of the disturbance. It shows that the response of the system has good tracking properties despite the presence of the disturbance. At the moment the disturbance occurs, the output curve exhibits a small overshoot, which is subdued in less than $2 \mathrm{~s}$.

The system operates in open-loop between two events, and we allow a relatively high tolerance $(\varepsilon=0.25)$. This explains the ripples that appear in the curve of the response. We can get rid of them by imposing stricter performance specifications (a smaller $\varepsilon$, and so, a smaller $\delta$ ). However, it means updating the control more often.

The bottom curve in Fig. 3 shows that the estimator can find the true value of the disturbance in a few time-steps. However,
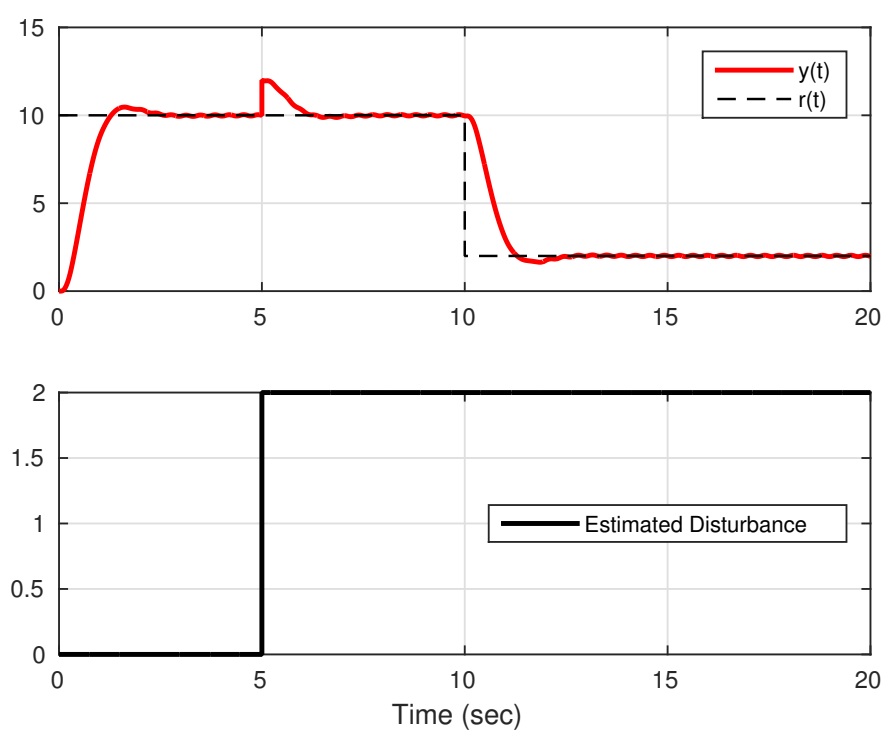

Figure 3. The output of the event-triggered system and the estimated disturbance

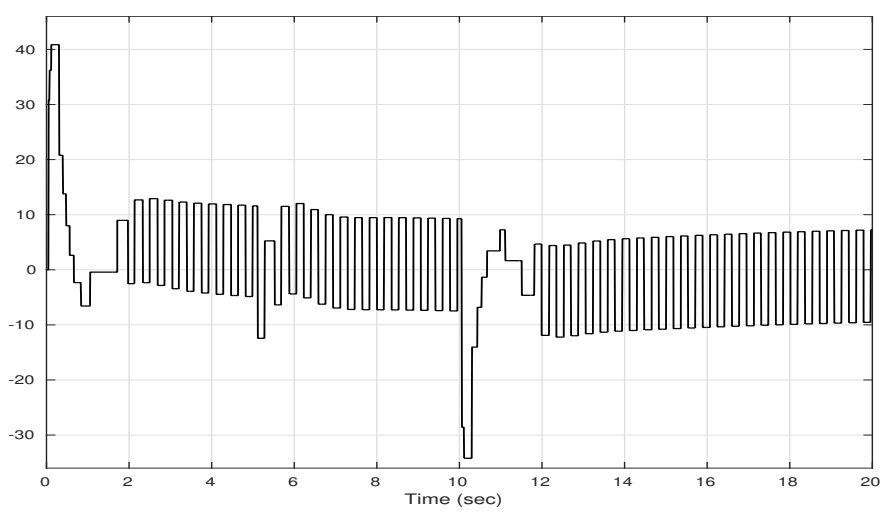

Figure 4. The event-triggered control law $\bar{u}(t)$

such good results are obtained on the condition that we provide the adequate initial values to the observer and the filter.

The high value of the tolerance $\varepsilon$ allowed us to compute and update the control law only 296 times in 200,000 sampling instants, as shown in Fig. 4. This proves that the communications between the controller and the actuators have been divided by a factor of more than 600 . Supposing that this communication relies on channels with a limited bandwidth, such channels will be largely relieved.

Fig. 5 shows the evolution of the Lyapunov-like function $V(e(t))$ in time. An event is triggered every time $V(e(t))$ reaches the threshold $\delta$, and $V(e(t))$ is pushed back inside the region enclosed by 0 and $\delta$. The graph shows that events are more frequent in transient intervals, where the control is updated more often to cope with the change in the set-point value. The events become sparser as the response settles to its final value.

Right after $t=5 \mathrm{~s}$, we notice an update of the control law but not an increase in the number of updates. This is due to the fact that the disturbance is estimated in one time-step and normal operation can quickly resume. 


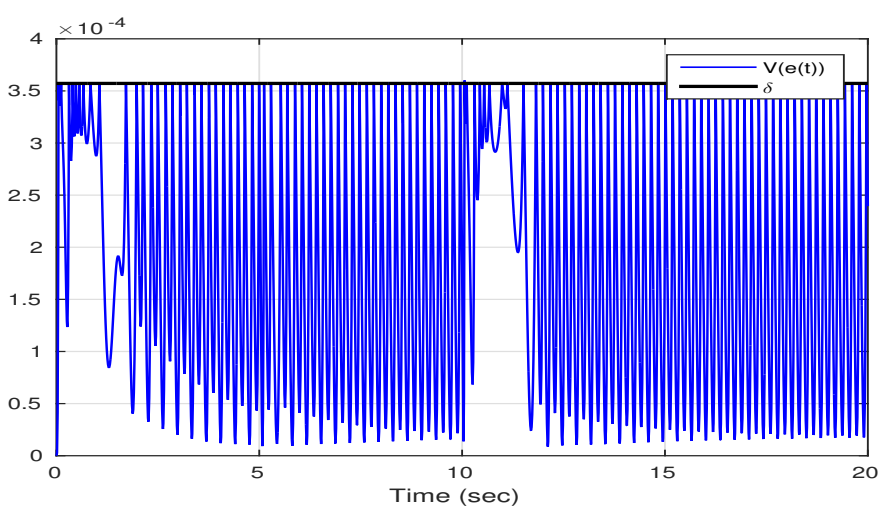

Figure 5. The Lyapunov-like function $V(e(t))$

\section{B. Sinusoidal disturbance}

The developed approach is tested a second time on a lowfrequency sinusoidal disturbance of the form

$$
d(t)=\cos (0.6 t) .
$$

The obtained response and the estimated disturbance are depicted in Fig. 6. It shows again that with only 272 updates (see Fig. 7) of the control law in 200,000 sampling instants, we manage to track the set-point. As shown in the top curve of Fig. 6 the effect of the disturbance on the output is attenuated with an about .1 factor. The reason for ending up with slightly fewer sampling instants resides in the fact that the cosine function is a continuous smooth function which is better handled than the abrupt-changing disturbance applied before. The estimator still manages to rapidly estimate the disturbance with a high accuracy.
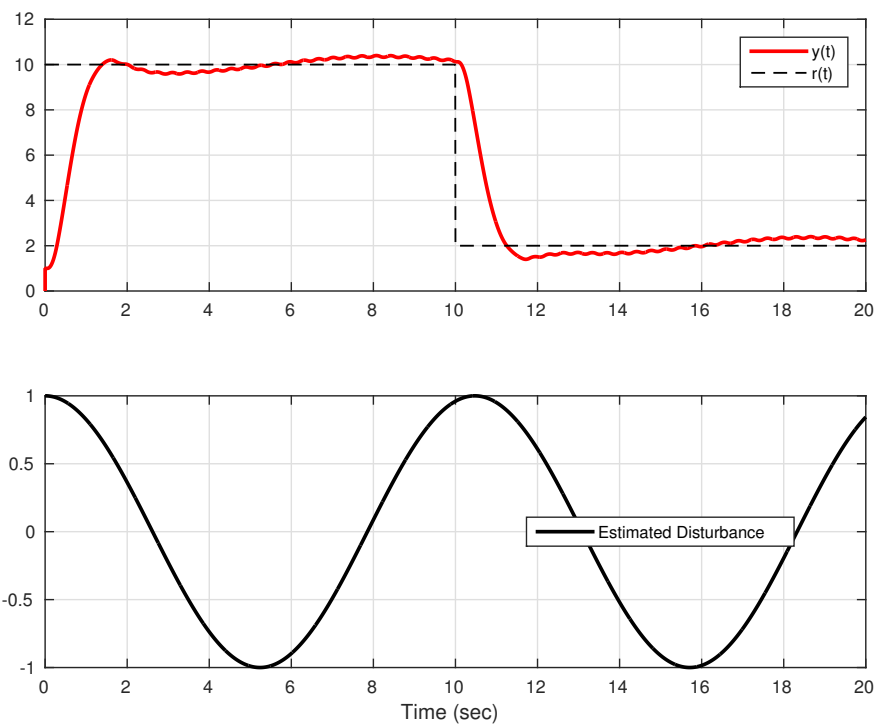

Figure 6. Response of the system under a sinusoidal disturbance and the estimated values of the disturbance

\section{CONCLUSION}

In this work, we have explored the behavior of the eventbased control approach in the presence of a load disturbance. Even though the value of the disturbance is unknown at first,

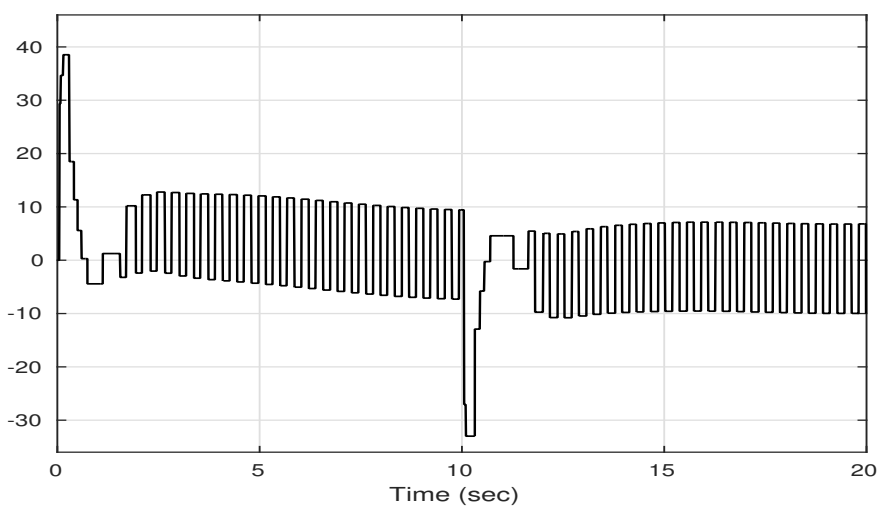

Figure 7. The control law $\bar{u}(t)$ under a sinusoidal disturbance

we have shown that we can accurately estimate it through the residual between the real output and an observed output. We have shown that if the appropriate initial conditions are provided to the estimator, the value of the disturbance is quickly found and given to the reference system. That way the work of the event-generator is not disturbed.

As a perspective for future work, we suggest to extend this approach to include other types of disturbances, on the input or the states of the system. Our ultimate goal is to be able to construct a self-triggered scheme, in which at times $t_{k}$ we compute the new control law and the time $t_{k+1}$ at which next event would occur. The need for checking the event-triggering conditions at every time instant would thus be removed.

\section{ACKNOWLEDGMENT}

This work has been partially supported by the LabEx PERSYVAL-Lab (ANR-11-61 LABX-0025-01).

\section{REFERENCES}

[1] K.-E. Årzén, "A simple event-based PID controller," in IFAC World Congress, Beijing, China, Jul. 1999.

[2] M. T. Donkers and W. M. Heemels, "Output-based event-triggered control with guaranteed $L^{\infty}$ gain and improved event-triggering," in 49th IEEE Conference on Decision and Control. Atlanta, GA, USA: IEEE, Dec. 2010, pp. 3246-3251.

[3] J. Lunze and D. Lehmann, "A state-feedback approach to event-based control," Automatica, vol. 46, no. 1, Jan. 2010, pp. 211-215.

[4] P. Tabuada, "Event-triggered real-time scheduling of stabilizing control tasks," IEEE Transactions on Automatic Control, vol. 52, no. 9, Sep. 2007, pp. 1680-1685.

[5] N. Marchand, S. Durand, and J. F. Guerrero Castellanos, "A general formula for event-based stabilization of nonlinear systems," IEEE Transactions on Automatic Control, vol. 58, no. 5, May 2013, pp. 13321337.

[6] N. Meslem and C. Prieur, "Event-based controller synthesis by bounding methods," European Journal of Control, vol. 26, Nov. 2015, pp. $12-21$.

[7] D. Lehmann and J. Lunze, "Event-based control: A state-feedback approach," in 2009 European Control Conference (ECC). IEEE, Aug. 2009, pp. 1716-1721.

[8] T. Ma, W. Cao, Y. Lin, and J. Zhang, "Observer-based event-triggered control with disturbance compensation," in 33rd Chinese Control Conference (CCC). Nanjing, China: IEEE, Jul. 2014, pp. 5602-5607.

[9] L. Etienne, S. Di Gennaro, and J.-P. Barbot, "Event triggered observerbased control for linear systems with time varying uncertainties," in American Control Conference. Chicago, IL, USA: IEEE, Jul. 2015, pp. 1531-1536. 
[10] J. Sun, J. Yang, S. Li, and W. X. Zheng, "Sampled-data-based eventtriggered active disturbance rejection control for disturbed systems in networked environment," IEEE Transactions on Cybernetics, vol. 49, no. 2, Feb. 2019, pp. 556-566.

[11] F. Zobiri, N. Meslem, and B. Bidégaray-Fesquet, "Event-based sampling algorithm for setpoint tracking using a state-feedback controller," in Second International Conference on Event-Based Control, Communications, and Signal Processing. Krakow, Poland: IEEE, Jun. 2016.

[12] S. X. Ding, Model-based Fault Diagnosis Techniques: Design Schemes, Algorithms, and Tools. Springer, 2008.

[13] W.-H. Chen, J. Yang, L. Guo, and S. Li, "Disturbance-observer-based control and related methods-an overview," IEEE Transactions on Industrial Electronics, vol. 63, no. 2, Feb. 2016, pp. 1083-1095.

[14] MathWorks. Aircraft pitch: System modeling. [Online]. Available: http://ctms.engin.umich.edu/CTMS/index.php?

example=AircraftPitch\&section=SystemModeling

\section{APPENDIX}

AROUND WOODBURY MATRIX IDENTITY

Combining (1)-(2) and (11)-(12), and defining $\tilde{x}(t)=$ $x(t)-\hat{x}(t)$, we obtain the following system

$$
\begin{aligned}
\dot{\tilde{x}}(t) & =(A-L C) \tilde{x}(t)-L d(t), \\
\tilde{y}(t) & =C \tilde{x}(t)+d(t) .
\end{aligned}
$$

This system is LTI and stable due to the Hurwitz nature of $A-L C$. Let $\mathcal{X}, \mathcal{Y}$ and $\mathcal{D}$ be the Laplace transforms of $\tilde{x}, \tilde{y}$ and $d$ respectively. Equations (22)-(23) yield

$$
\begin{aligned}
& \mathcal{X}(s)=-(s I-(A-L C))^{-1} L \mathcal{D}(s), \\
& \mathcal{Y}(s)=C \mathcal{X}(s)+\mathcal{D}(s) .
\end{aligned}
$$

where $I$ is the identity matrix, and hence

$$
\mathcal{Y}(s)=\left(I-C(s I-(A-L C))^{-1} L\right) \mathcal{D}(s) .
$$

This allows to first write $\mathcal{D}(s)$ in terms of $\mathcal{Y}(s)$ :

$$
\mathcal{D}(s)=\left(I-C(s I-(A-L C))^{-1} L\right)^{-1} \mathcal{Y}(s) .
$$

We can compute the inverse using Woodbury matrix identity.

Lemma 1 (Woodbury matrix identity): Let $\mathrm{A}$ and $\mathrm{B}$ be two invertible matrices and $\mathrm{U}$ and $\mathrm{V}$ two matrices with matching dimensions then

$$
(\mathrm{A}+\mathrm{UBV})^{-1}=\mathrm{A}^{-1}-\mathrm{A}^{-1} \mathrm{U}\left(\mathrm{B}^{-1}+\mathrm{VA}^{-1} \mathrm{U}\right)^{-1} \mathrm{VA}^{-1} \text {. }
$$

We apply Lemma 1 with $\mathrm{A}=I, \mathrm{U}=C, \mathrm{~V}=L$ and $\mathrm{B}=-(s I-A+L C)^{-1}$ to obtain

$$
\mathcal{D}(s)=\left(I+C(s I-A)^{-1} L\right) \mathcal{Y}(s) .
$$

We now go back to the time space and find that this equation is equivalent to solving the system

$$
\begin{aligned}
\dot{m}(t) & =A m(t)+L \tilde{y}(t), \\
\hat{d}(t) & =C m(t)+\tilde{y}(t) .
\end{aligned}
$$

where $m$ is merely an auxiliary variable with no specific interpretation. 